Esta publicación cientifica en formato digital es continuidad de la revista impresa ISSN-Versión Impresa 0798-1406 / ISSN-Versión on line 2542-3185Depósito legal pp


Instituto de Estudios Políticos y Derecho Público "Dr. Humberto J. La Roche" de la Facultad de Ciencias Jurídicas y Políticas de la Universidad del Zulia Maracaibo, Venezuela
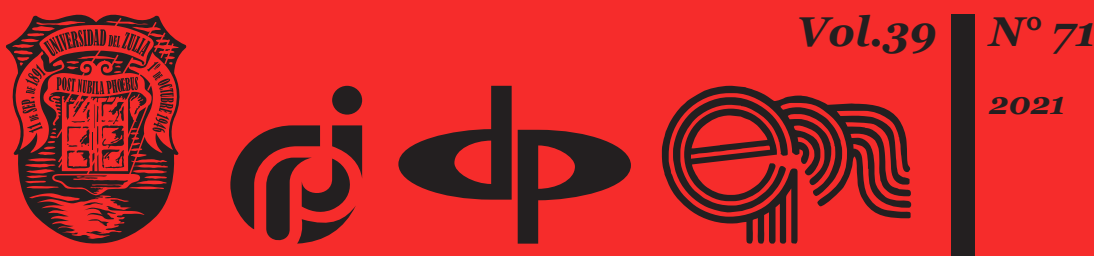


\title{
Escasa eficiencia en costos y plazos en proyectos de inversión en Arequipa y Moquegua 2004-2020
}

\author{
DOI: https://doi.org/10.46398/cuestpol.3971.49
}

\author{
Javier Pedro Flores Arocutipa * \\ Jorge Jinchuña Huallpa ** \\ Rocío Claribel Cornelio Aira *** \\ Moisés Chacolla Soto $* * * *$ \\ José Miguel de la Paz Ramos ***** \\ Luís Enrique Fernández Sosa ${ }^{* * * * * *}$
}

\section{Resumen}

Objetivo: Demostrar la escasa eficiencia en costos y plazos en 22 proyectos de inversión (PIP) del Gobierno Regional de Moquegua y Arequipa, los cuales generaron mayores costos y plazos entre el ex ante y el ex post en el periodo 2004-2020. Método: Inductivo, básico, no experimental. Resultados: En base a la prueba de Wilcoxon, se demostró que los costos de 12 proyectos en Moquegua, antes y después, fueron diferentes con significancia asintótica (bilateral) 0.006, de 127 a 220 millones; y la prueba " $\mathrm{t}$ " para muestras relacionadas generó la significancia (bilateral) de o.ooo. Se observó que la media calculada en días fue diferente, que la media del plazo ex ante resultó ser de 339 días mientras que la media del plazo real fue de 2307 días. En 10 proyectos de Arequipa, la prueba de Wilcoxon generó 0.007 y los costos pasaron de 1030 a 1585 millones de soles; la diferencia de plazos generó un p-valor de wilcoxon de 0.004 de una media de 15.4 a 57.5 meses. Conclusión: Los costos del periodo anterior y posterior son diferentes; al igual que los plazos propuestos y los plazos efectivos en ambas regiones.

\footnotetext{
* Doctor en Administración, Doctor en Economía, Magister en Gestión Pública, Abogado. Economista, Ingeniero Comercial. Docente escuela de post grado, Universidad Nacional de San Agustín, Perú. ORCID ID: https://orcid.org/oooo-0003-0784-4153

** Doctor en Ciencias de la Educación, Maestro en Ciencias (Magister Scientiae) en Contabilidad Auditoria, Contador Público. Docente de la Universidad Nacional de Moquegua, Perú. ORCID ID: https://orcid. org/oooo-0oo2-9073-3798

*** Ingeniero Comercial con Maestría en Gestión Pública, Docente de la Universidad José Carlos Mariátegui. Moquegua, Perú. ORCID ID: https://orcid.org/oooo-0003-4984-9550

**** Doctor en Comunicación y Desarrollo. Profesor de lengua, literatura y gestión educativa. Docente de la Universidad Nacional Jorge Basadre Grohmann de Tacna, Perú. ORCID ID: https://orcid.org/ooooooo3-3397-1199

***** Maestro en Gestión Pública, Ingeniero Comercial, Docente de la Universidad José Carlos Mariátegui. Moquegua, Perú. ORCID ID: https://orcid.org/oooo-00o3-1096-1457

****** Maestro en Ciencias (Magister Scientiae) en Contabilidad Auditoria, Contador Público, Universidad Nacional de Moquegua, Perú. ORCID ID: https://orcid.org/oooo-0001-5707-296X
}

Recibido el 15/07/2021 Aceptado el 28/10/2021 
Palabras claves: escasa eficiencia; costos; plazos; proyectos de inversión; corrupción administrativa.

\title{
Low cost and time efficiency in investment projects in Arequipa and Moquegua 2004 - 2020
}

\begin{abstract}
Objective: To demonstrate the low efficiency in costs and timeframes in 22 investment projects (PIP) of the Regional Government of Moquegua and Arequipa, which generated higher costs and timeframes between the ex ante and ex post in the period 2004-2020. Method: Inductive, basic, not experimental. Results: Based on the Wilcoxon test, it was shown that the costs of 12 projects in Moquegua, before and after, were different with asymptotic significance (bilateral) 0.006, from 127 to 220 million; and the " $\mathrm{t}$ " test for related samples generated the significance (bilateral) of o.ooo. It was observed that the average calculated in days was different, that the average of the ex ante period turned out to be 339 days while the average of the actual period was 2307 days. In 10 projects in Arequipa, the Wilcoxon test generated 0.007 and the costs went from 1030 to 1585 million soles; the difference in terms generated a p-value of wilcoxon of 0.004 from an average of 15.4 to 57.5 months. Conclusion: The costs of the previous and subsequent period are different, as are the proposed deadlines and actual deadlines in both regions.
\end{abstract}

Keywords: Low efficiency; costs; time frame; investment projects; administrative corruption.

\section{Introducción}

Los proyectos deinversión pública (PIP) van dela mano con el crecimiento económico y la consolidación del capital institucional. La necesidad de una buena gestión de proyectos es relevante y se explica por múltiples factores, doce son los que señala (Villegas, 2018). Los planes estratégicos, la gestión por resultados se vuelven verso frente a la realidad de perjuicios contra la nación que descubre la contraloría. Los 22 mil millones de perjuicio al estado empiezan en los proyectos. Un factor es el alargamiento de los plazos y el otro, los sobrecostos, se nota cuando los costos del proyecto viable distan del proyecto ejecutado. Así mismo, con los plazos. 
Javier Pedro Flores Arocutipa, Jorge Jinchuña Huallpa, Rocío Claribel Cornelio Aira, Moisés Chacolla Soto, José Miguel de la Paz Ramos y Luís Enrique Fernández Sosa

El capital y el trabajo se combinan: el primero impulsa al segundo y viceversa. El porcentaje de crecimiento de la renta por persona es resultado de la productividad del trabajo que se consigue por el incremento de la tecnología (Duarte \& Ruíz, 2015). Entonces, ¿̇por qué no se ejecutan en su momento los proyectos de inversión? En este sentido Balcázar (2015), citado por (Fernández, 2018), afirma que los problemas de titularidad de propiedades, pésimos perfiles de proyectos y la falta de saneamiento de los terrenos donde se pretende construir repercuten en la demora en los permisos; de la misma manera, influyen en que los tiempos y costos transcurran en perjuicio del costo de oportunidad de los recursos.

Arela Bobadilla (2020) sostiene que son proyectos (PIP) de los sectores, agricultura y transportes donde hay mayores retrasos. Asimismo, Romero (2016) afirma que los proyectos (PIP) de los sectores, agricultura y transporte superan los estándares de costos en la ejecución. La Contraloría, en el informe sobre el cálculo de la corrupción en el Perú, señala que los sectores de transporte, educación y salud son los que mayormente incumplen con los plazos (Shack et al., 2020)pues los costos de ineficiencia que generan este tipo de conductas, muchas veces, están relacionados con la introducción de grandes distorsiones $\mathrm{y}$, por consiguiente, con la falta de alineación de las decisiones políticas con una actitud íntegra y actuación eficiente del Estado. El primer paso para determinar los efectos de la corrupción es, sin duda, la identificación del tamaño de la misma. En tal sentido, a partir de la exploración de los productos del control gubernamental, como la base de un modelo experimental natural de observación directa de la corrupción, este trabajo propone una metodología de cálculo del tamaño de este fenómeno. En otras palabras, sobre la base de la extrapolación del perjuicio económico producido al Estado (i.e. sobrevaloraciones, pagos injustificados, entre otros hechos de similar importancia. Al respecto señala que es el $15 \%$ del presupuesto público ejecutado que se pierde por los delitos contra la administración pública, por corrupción e inconducta funcional.

Ahora la fiscalía observa que se constituyen organizaciones criminales constituidas para robarle al estado. Esto es importante saber, los sobrecostos al estado, por ejemplo, en la ejecución de una obra pública, se requiere que se coludan -como mínimo- el funcionario público que da la conformidad del sobre valor, el que supervisa y firma el sobre valor y el Ing. residente y contratista que presentan los documentos sobrevalorados. Y ello ocurre cuando el estado pretende adquirir un bien, se otorga un servicio o adjudique una obra. ¿Tiene algo que ver la dimensión de lo que se le paga (soborno) al funcionario o al jefe de pliego? Claro, es el tamaño del daño causado al presupuesto público. Lo que se pierde por 10 debido a que la coima es el diezmo.

Quién no ha escuchado del Caso Lavajato, que captaba a presidentes, ministros, gobernadores, alcaldes políticos en campaña (Proetica, 2021) 
cuyo fin era llegar al poder del estado y al presupuesto público para delinquir. Allí está también la "centralita" en Ancash, el caso Manos limpias, en Chiclayo. y cuál es el perjuicio que la corrupción le causa al Perú y las regiones? Según estimaciones de Nelson Schak, (Shack et al., 2020) pues los costos de ineficiencia que generan este tipo de conductas, muchas veces, están relacionados con la introducción de grandes distorsiones $\mathrm{y}$, por consiguiente, con la falta de alineación de las decisiones políticas con una actitud íntegra y actuación eficiente del Estado. El primer paso para determinar los efectos de la corrupción es, sin duda, la identificación del tamaño de la misma. En tal sentido, a partir de la exploración de los productos del control gubernamental, como la base de un modelo experimental natural de observación directa de la corrupción, este trabajo propone una metodología de cálculo del tamaño de este fenómeno. En otras palabras, sobre la base de la extrapolación del perjuicio económico producido al Estado (i.e. sobrevaloraciones, pagos injustificados, entre otros hechos de similar importancia de Alfonso Quiroz (Blondet, 2013), en los últimos años es un promedio anual estimado del $3 \%$ a $4 \%$ de su Producto Bruto Interno (PBI).

Así en el caso de las economías regionales de Arequipa seria de 900 millones de soles anuales y de Moquegua sería un aproximado de 240 millones de soles anuales. Es una pena decirlo, Moquegua y Tumbes lideran con funcionarios por cada 100 mil habitantes con denuncias administrativas, civiles y penales por presuntas faltas y delitos cometidos en sus funciones, sea colusión, peculado, malversación o negociación incompatible. Así Moquegua tiene 62 denunciados por cada 100 mil habitantes, Tumbes 61 , Tacna 50 denunciados. Arequipa 17 de cada 100 mil. Calculando el 3\% del PBI regional y multiplicando por 10 años, los resultados se muestran en la Tabla 1 y en la Figura 1.

\section{Tabla 1. Pérdidas generadas por la corrupción por pliegos}

\begin{tabular}{lll}
\hline Sector & Pérdidas 2020 & Porcentaje \\
\hline Gobierno Nacional & $11,580,217,388$ & $10.3 \%$ \\
Gobierno Regional & $5,762,725,383$ & $15 \cdot 7 \%$ \\
Gobierno Local & $4,716,240,287$ & $17.6 \%$ \\
Transportes y comunicaciones & $1,515,916,802$ & \\
Educación & $1,546,657,991$ & \\
\hline
\end{tabular}

Fuente: Contraloría general de la república del Perú. 
Javier Pedro Flores Arocutipa, Jorge Jinchuña Huallpa, Rocío Claribel Cornelio Aira, Moisés Chacolla Soto, José Miguel de la Paz Ramos y Luís Enrique Fernández Sosa

804 Escasa eficiencia en costos y plazos en proyectos de inversión en Arequipa y Moquegua 2004-2020

\section{Figura 1. La corrupción en la última década en el sur Peruano} (2011 - 2020) en soles

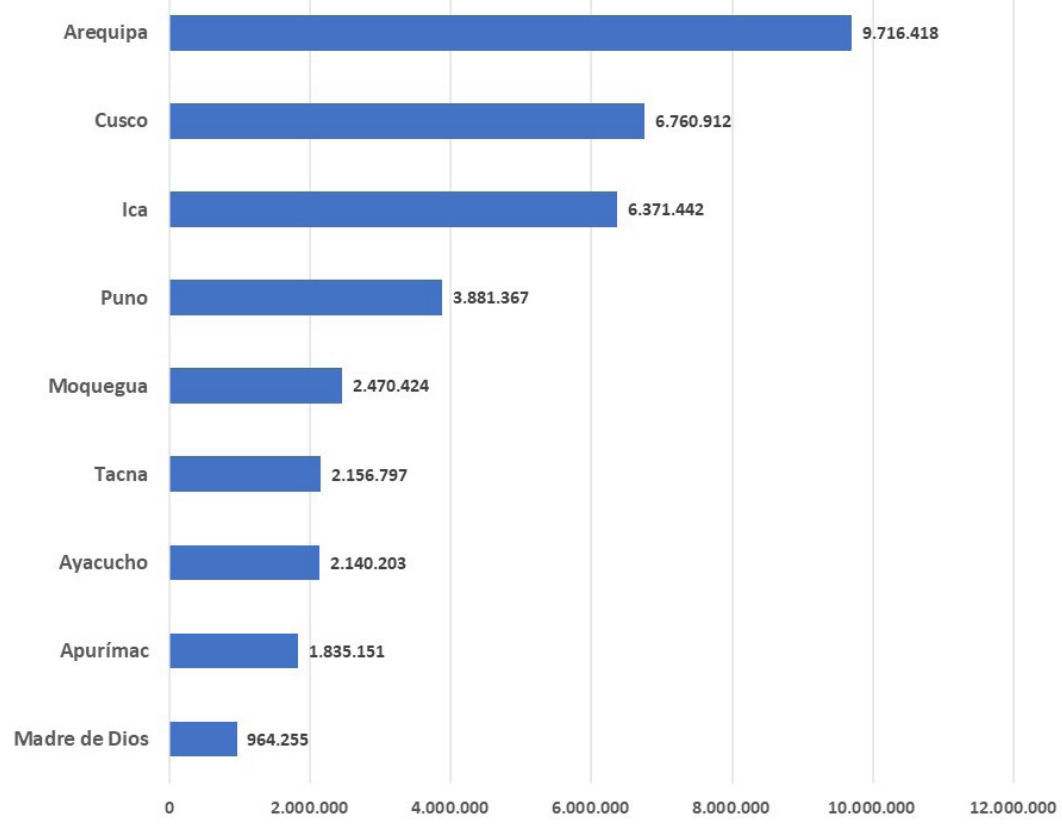

Fuente: Elaboración propia con información de Contraloría de la república del Perú.

Otro fáctico es el generado por el Instituto Nacional de Estadística e Informática INEI (INEI, 2021), cuando el 3.7\% de los respondientes en el Perú dijeron que tuvieron que pagar, entiéndase sobornar, por petición, por solicitar un servicio en instituciones públicas. Para la encuestadora (Proetica, 2021) el 13\% de los respondientes aseguran que tuvieron que pagar coimas. Y en el índice del Barómetro de las Américas (Latino barómetro, 2021) se afirma que un $26.3 \%$ de personas fue víctima de la corrupción, pero según proética existe una alta tasa, del 91\%, que no realiza las denuncias porque no confía en las autoridades, fiscalía, poder judicial, contraloría, policía nacional, dicen que no encontraran justicia. respecto el debido proceso (Sánchez, 2020), refiere que cada país lo ha adecuado a sus exigencias, un debido proceso aplicable tanto a la vía administrativa como a la judicial.

Burgos \& Vela, (2015)multidrug resistanceassociated protein 1 (MRP1/ ABCC1 destacan la importancia de la programación y monitoreo de las obras, dado que es la única manera de identificar fallas en los procesos técnicos 
como administrativos, y con esto se evitarían sobrecostos e incumplimiento de plazos. De hecho, el requisito básico es cumplir con la programación de los PIP, y eso, al parecer, no se cumple en el Gobierno Regional de Arequipa (en adelante GRA) y Gobierno Regional de Moquegua (en adelante GRM). (Gordo et al., 2017) señalan que la vida útil de un proyecto está destinada al éxito o al fracaso desde el momento de su concepción, pues desde que se elabora el perfil puede empezar el "favorecimiento" o "direccionalidad". También, se ha observado que, desde la elaboración de referencias, se favorecen marcas y proveedores.

Porras \& Diaz (2015) sostienen que, con un correcto cálculo de los costos del PIP se evita sobrecostos y/o asignación de menor presupuesto del requerido. Huaquisto (2016) afirma que, a menor eficiencia de la supervisión del PIP, mayores son los costos y retrasos. Por su parte, Dueñas (2017) vinculó el mayor beneficio social (valor actual neto social) con el óptimo de costos y plazos del PIP.

Para iniciar u operar un proyecto, es ideal lograr el menor costo de los factores de producción. Esta es una ley del capital. En nuestro caso es el tamaño del PIP relacionados con la tecnología y los costos. Se trata de encontrar una relación óptima de factores que permita ubicar un máximo de beneficio social a un costo óptimo. Usualmente son capital (K) y trabajo (L). El tiempo está contenido en el capital.

La liquidación de obras, el cierre de PIP y la evaluación ex post; que es una debilidad en los estados regionales, como menciona (Beltrán, 2010), deberían cumplirse, precisamente, para corregir defectos y lograr mayor beneficio social.

Se ha observado en Moquegua y Arequipa, específicamente en sus gobiernos subnacionales, la programación de diferentes proyectos. Al final de cada periodo, algunos resultan ejecutados, otros no. También se ha notado que hay proyectos que se vienen ejecutando en varios periodos anuales y que superan su plazo de ejecución en meses, o incluso en años. Esto por supuesto lleva a la percepción de lesión del presupuesto público por incremento de costo y plazos. La pretensión de la investigación, es saber cuántos proyectos emblemáticos programados para un determinado plazo y costo terminan siendo mayores; cuántos proyectos tienen mayor avance, y; cuáles son los proyectos que se vienen ejecutando en varios periodos, al notar que los beneficios sociales se demoran y los costos se incrementan. Por ello, en esta primera parte, se genera una visión panorámica y ejecutiva de la situación de los proyectos del Gobierno Subnacional de Moquegua (MEF - DGPMI, 2021). 
Javier Pedro Flores Arocutipa, Jorge Jinchuña Huallpa, Rocío Claribel Cornelio Aira, Moisés Chacolla Soto, José Miguel de la Paz Ramos y Luís Enrique Fernández Sosa

Figura 2. Proyectos programados por años en el Gobierno Regional de Moquegua 2004 - 2019

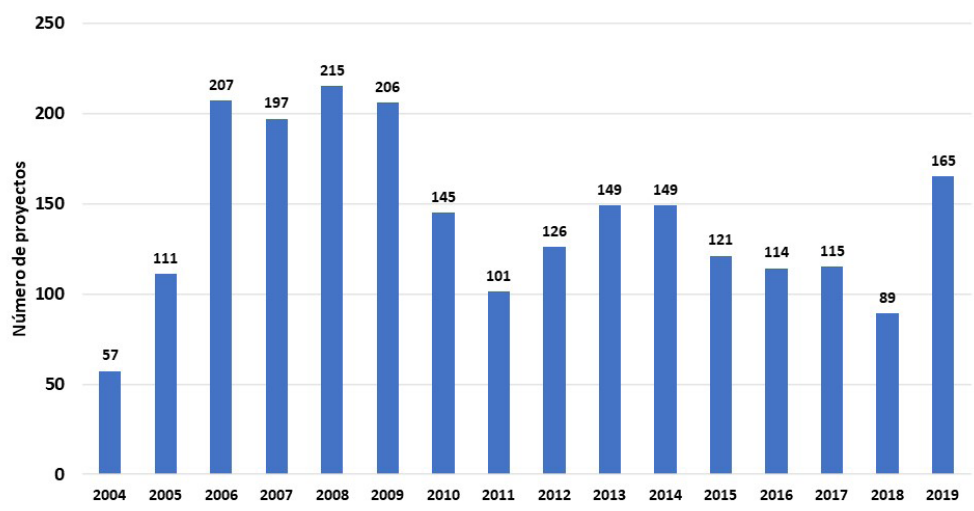

Fuente: Elaboración propia.

En la Figura 2, sobre los proyectos programados por años en el GRM 2004-2019, se muestra un mínimo de 57 proyectos y un máximo de 215. En la etapa 2006 y 2009 fue el máximo de proyectos programados y en el periodo 2015 y 2018, el mínimo; en todos los años hubo proyectos que no se ejecutaron. En ese sentido, existen en promedio 31 proyectos (MEF DGPMI, 2019).

Figura 3: Representación porcentual de 15 proyectos emblemáticos por año del Gobierno Regional de Moquegua

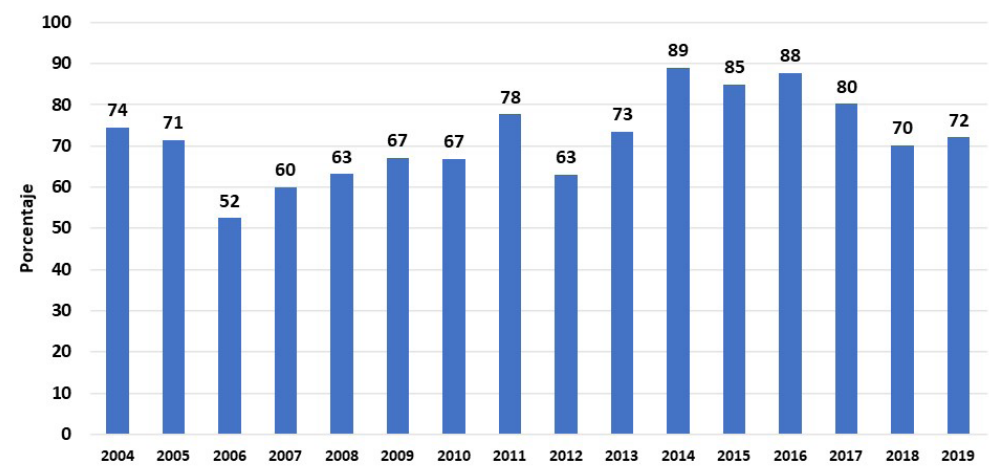

Fuente: Elaboración propia. 
En la Figura 3, los 15 proyectos representan el porcentaje que se indica. Así, los 15 proyectos emblemáticos son los que ejecutó el GRM. El PIP, con código 2017318, indica una demora que figura entre los años 2004 al año 2017 (MEF - DGPMI, 2021).

Tabla 2. Proyectos emblemáticos de la región Moquegua

\begin{tabular}{|c|c|c|c|c|}
\hline Devengado & $\begin{array}{l}\text { Código: } \\
\text { 2017318: } \\
\text { reubicación } \\
\text { y amplia- } \\
\text { ción del } \\
\text { PTAR- Mo- } \\
\text { quegua }\end{array}$ & $\begin{array}{c}\text { Código: } \\
\text { 2o46177: } \\
\text { mejoramien- } \\
\text { to de red vial } \\
\text { departamen- } \\
\text { tal Moque- } \\
\text { gua-Arequi- } \\
\text { pa }\end{array}$ & $\begin{array}{c}\text { Código: } \\
\text { 2057931: } \\
\text { Amp. y } \\
\text { mejora del } \\
\text { hospital de } \\
\text { Moquegua }\end{array}$ & $\begin{array}{c}\text { Código: } \\
\text { 2022049: } \\
\text { represa Chi- } \\
\text { rimayuni, } \\
\text { Chojata y } \\
\text { Lloque }\end{array}$ \\
\hline 2019 & & $4,051,111$ & $7,546,525$ & 38,664 \\
\hline 2018 & & $15,616,336$ & $14,237,852$ & 29,842 \\
\hline 2017 & 834,696 & $9,842,439$ & $70,143,555$ & 193,952 \\
\hline 2016 & 22,500 & $4,929,657$ & $58,425,209$ & 155,395 \\
\hline 2015 & 208,426 & $12,939,383$ & $32,988,434$ & 208,117 \\
\hline 2014 & $1,154,846$ & $51,576,516$ & $54,546,554$ & $2,356,723$ \\
\hline 2013 & $1,360,712$ & $35,547,144$ & $27,559,091$ & $7,030,956$ \\
\hline 2012 & $2,820,415$ & 456,050 & $1,089,562$ & $14,547,135$ \\
\hline 2011 & $7,956,422$ & o & o & $6,730,880$ \\
\hline 2010 & $9,946,750$ & o & 69,460 & 1,340 \\
\hline 2009 & $18,419,902$ & $\mathrm{O}$ & 302,050 & 78,694 \\
\hline 2008 & $5,207,287$ & 5,574 & 439,872 & 10,530 \\
\hline 2007 & $3,592,981$ & 248,967 & o & 60,849 \\
\hline 2006 & 776,279 & $\mathrm{O}$ & o & 80 \\
\hline 2005 & 434,019 & $\mathrm{O}$ & o & o \\
\hline 2004 & 407,377 & o & $\mathrm{O}$ & 0 \\
\hline TOTAL & $53,142,612$ & 135,213,177 & $267,348,164$ & $31,443,157$ \\
\hline
\end{tabular}

Fuente: Elaboración propia.

La Tabla 2, señala que el proyecto con código 2046177, conocido como la carretera Moquegua - Arequipa, se invirtieron un total de 135.2 millones de soles y al 2019 fueron 10 años desde su inicio. El proyecto 2057931 ha devengado 267.3 millones de soles, por lo que son 11 años con devengados. 
Javier Pedro Flores Arocutipa, Jorge Jinchuña Huallpa, Rocío Claribel Cornelio Aira, Moisés Chacolla Soto, José Miguel de la Paz Ramos y Luís Enrique Fernández Sosa

808 Escasa eficiencia en costos y plazos en proyectos de inversión en Arequipa y Moquegua 2004-2020

En el caso del código 2022049, este cuenta con 14 años de ejecución (MEF - DGPMI, 2021).

Tabla 3. Proyectos emblemáticos de la región Moquegua

\begin{tabular}{|c|c|c|c|c|c|c|}
\hline $\begin{array}{c}\text { Deven- } \\
\text { gado }\end{array}$ & $\begin{array}{l}\text { 2109460: } \\
\text { Infraes- } \\
\text { tructura } \\
\text { del ISTU- } \\
\text { JCM }\end{array}$ & $\begin{array}{l}\text { 2159754: } \\
\text { Centro } \\
\text { de trata- } \\
\text { miento } \\
\text { enferme- } \\
\text { dades }\end{array}$ & $\begin{array}{l}\text { 2166595: } \\
\text { Amp. de la } \\
\text { frontera } \\
\text { agricola } \\
\text { Lomas de } \\
\text { Ilo }\end{array}$ & $\begin{array}{c}\text { 2o87465: } \\
\text { red vial } \\
\text { mo-534, } \\
\text { San Ge- } \\
\text { rónimo } \\
\text { - el Alga- } \\
\text { rrobal }\end{array}$ & $\begin{array}{l}\text { 2159756: } \\
\text { Prog.no } \\
\text { escola- } \\
\text { rizados } \\
\text { de edu- } \\
\text { cación } \\
\text { inicial }\end{array}$ & $\begin{array}{c}\text { 2091434: } \\
\text { Accesos } \\
\text { a la irri- } \\
\text { gación } \\
\text { Pampas } \\
\text { San An- } \\
\text { tonio }\end{array}$ \\
\hline 2020 & 27,077 & o & 185,935 & $2,658,991$ & 220,420 & o \\
\hline 2019 & $7,752,779$ & 710,765 & $17,476,373$ & $2,204,166$ & $1,903,813$ & o \\
\hline 2018 & $8,389,367$ & $2,327,469$ & 727,370 & $2,290,823$ & $1,500,998$ & o \\
\hline 2017 & $8,239,222$ & $2,974,692$ & 978,344 & 378,324 & $1,010,145$ & $\mathrm{O}$ \\
\hline 2016 & $12,891,464$ & $1,712,452$ & $2,060,617$ & 250,581 & 328,769 & 346,334 \\
\hline 2015 & $3,452,850$ & $3,432,988$ & $17,864,092$ & $2,554,822$ & 457,410 & $5,535,751$ \\
\hline 2014 & $1,304,557$ & $2,597,190$ & $107,133,933$ & $8,094,172$ & $\mathrm{O}$ & 4,076,095 \\
\hline 2013 & $7,989,937$ & $1,758,777$ & $38,620,879$ & 970,182 & o & o \\
\hline 2012 & $5,586,274$ & 2,500 & o & 33,029 & o & 149,678 \\
\hline 2011 & $3,798,719$ & o & 0 & 102,713 & 0 & o \\
\hline 2010 & $1,182,191$ & o & o & o & $\mathrm{O}$ & $\mathrm{O}$ \\
\hline TOTAL & $60,614,437$ & $15,516,833$ & $\mathbf{1 8 5}, \mathbf{0 4 7 , 5 4 3}$ & 19,537,803 & $5,421,555$ & $10,107,858$ \\
\hline
\end{tabular}

Fuente: Elaboración propia.

La Tabla 3 corrobora que el PIP código 2109460, al 2020, suma 11 años. El PI, código 2159754, al 2020 tiene 9 años de inversiones. El PIP con código 2166595, Lomas de ILO, tiene 8 años; el proyecto 2166595, 8 años. El PIP 2087465 de San Gerónimo, cuenta con 10 años. El PIP 2159756, Programa No Escolarizados de Educación Inicial, dura a la fecha seis años, mientras el proyecto 2091434 duró cinco años. 
Tabla 4. Proyectos emblemáticos de la región Moquegua

\begin{tabular}{cccccc}
\hline Devengado & $\begin{array}{c}\text { 2031201: } \\
\text { el Pampa } \\
\text { Jaguay } \\
\text { rinconada }\end{array}$ & $\begin{array}{c}\mathbf{2 1 1 6 0 5 8 :} \\
\text { electrifica- } \\
\text { ción rural } \\
\text { MN GSC }\end{array}$ & $\begin{array}{c}\text { 2112687: } \\
\text { construc- } \\
\text { ción del } \\
\text { almacén } \\
\text { de medi- } \\
\text { camentos }\end{array}$ & $\begin{array}{c}\mathbf{2 1 5 9 7 5 5 :} \\
\text { servicios } \\
\text { de salud } \\
\text { sexual y } \\
\text { reproduc- } \\
\text { tiva }\end{array}$ & $\begin{array}{c}\text { IE coronel } \\
\text { Fco. } \\
\text { Bolognesi } \\
\text { Cervantes, } \\
\text { Ilo }\end{array}$ \\
\hline 2019 & 9,251 & & 393,396 & 0 & 134,183 \\
2018 & $2,235,827$ & & 41,751 & 680,333 & $1,983,360$ \\
2017 & $2,201,868$ & 5,000 & 27,725 & $3,016,567$ & $5,535,018$ \\
2016 & $6,276,814$ & 22,159 & 17,423 & 151,909 & $3,223,688$ \\
2015 & $18,901,581$ & $1,528,106$ & 0 & 292,741 & $3,557,543$ \\
2014 & $4,864,097$ & $3,944,338$ & 0 & 338,718 & $2,516,213$ \\
2013 & $\mathbf{1 , 4 0 6 , 5 2 6}$ & $\mathbf{1 1 6 , 4 3 6}$ & 40,692 & 665,338 & 315,048 \\
2012 & 0 & 1,962 & 520,987 & 34,986 & 35,799 \\
2011 & 0 & 70,521 & 0 & 0 & 0 \\
TOTAL & $\mathbf{3 5 , 8 9 5 , 9 6 4}$ & $\mathbf{5 , 6 8 8 , 5 2 2}$ & $\mathbf{1 , 1 3 2 , 3 6 4}$ & $\mathbf{5 , 1 8 0 , 5 9 2}$ & $\mathbf{1 7 , 3 0 0 , 8 5 2}$ \\
\hline
\end{tabular}

Fuente: Elaboración propia.

La Tabla 4 muestra el proyecto 2031201, cuyo tiempo de ejecución viene siendo de siete años. El PIP 2116058 demandó siete años, 2013 al 2019. En el caso del proyecto 2112687, Construcción del Almacén de Medicamentos, han pasado 8 años y continúa en ejecución. El PIP 2159755 tuvo una duración efectiva de siete años. Por último, el proyecto de mejoramiento 2160741 a la fecha tiene una duración de 9 años.

Tabla 5. Proyectos emblemáticos de la región Moquegua

\begin{tabular}{cccccccc}
\hline & $\begin{array}{c}\text { 2164210: } \\
\text { casa ho- } \\
\text { gar(car) } \\
\text { para } \\
\text { niños en } \\
\text { abando- } \\
\text { no }\end{array}$ & $\begin{array}{c}\text { 2166593: } \\
\text { radiación } \\
\text { UV nivel } \\
\text { primario } \\
\text { Ia UGEL- } \\
\text { ILO. }\end{array}$ & $\begin{array}{c}\text { 2167234: } \\
\text { radiación } \\
\text { UGV en } \\
\text { UGEL ILO }\end{array}$ & $\begin{array}{c}\text { 2173528: } \\
\text { servicio } \\
\text { de } \\
\text { energía } \\
\text { eléctrica } \\
\text { Ilo }\end{array}$ & $\begin{array}{c}\text { 2177187: } \\
\text { carretera } \\
\text { a nivel } \\
\text { de trocha } \\
\text { carrozable, }\end{array}$ & $\begin{array}{c}\text { 2177741: } \\
\text { TIC en } \\
\text { II.EE. de } \\
\text { UGEL } \\
\text { MN. GSC. }\end{array}$ & $\begin{array}{c}\text { 2187790: } \\
\text { cadenas } \\
\text { producti- } \\
\text { vas agrí- } \\
\text { colas }\end{array}$ \\
\hline 2019 & $1,168,976$ & 98,447 & $1,341,745$ & 125,730 & $8,162,531$ & 151,865 & 728,804 \\
2018 & 958,192 & $1,786,136$ & $2,326,357$ & 769,370 & $11,020,501$ & 302,188 & 564,084 \\
2014 & 145,438 & 476,938 & $2,855,655$ & $2,282,612$ & $11,882,559$ & 382,568 & 654,824 \\
& & & & & & & \\
\hline
\end{tabular}




\begin{tabular}{|c|c|c|c|c|c|c|c|}
\hline \multirow{2}{*}{$\begin{array}{r}810 \\
2016\end{array}$} & \multicolumn{7}{|c|}{$\begin{array}{l}\text { Javier Pedro Flores Arocutipa, Jorge Jinchuña Huallpa, Rocío Claribel Cornelio Aira, Moisés } \\
\text { Chacolla Soto, José Miguel de la Paz Ramos y Luís Enrique Fernández Sosa }\end{array}$} \\
\hline & $2,101,535$ & $2,415,401$ & & o & $3,237,728$ & 697,834 & 697,578 \\
\hline 2015 & 490,534 & $1,488,785$ & & o & $3,659,755$ & 656,940 & $1,025,919$ \\
\hline 2014 & 35,039 & 112,571 & 157,309 & 4,000 & $1,441,448$ & 424,331 & 95,000 \\
\hline 2013 & 1,300 & 91,232 & 107,869 & 88,493 & 402,241 & 418,935 & o \\
\hline & $6,120,813$ & $6,469,510$ & $6,788,935$ & $3,270,205$ & $39,806,763$ & $3,034,661$ & $3,766,209$ \\
\hline
\end{tabular}

Fuente: Elaboración propia.

La Tabla 5 muestra que el proyecto 2164210 tiene una duración de 8 años, incluido el Presupuesto Institucional Modificado del año 2020. El proyecto 2166593 dura ocho años, puesto que en el año 2020 se han destinado 70 mil soles al mismo. El proyecto 2167234 tiene siete años desde que empezó. El PIP 2173528 acumula siete años y 84 meses. El PIP 2177187, un proyecto de carretera carrozable comunica Quinistaquillas a Cuchumbaya, dura siete años. El Devengado del proyecto con código 2177741 trata de las TIC; también con siete años. Los años de ejecución del proyecto 2187790 van por siete años desde el año 2014 (MEF - DGPMI, 2021).

Ante lo expuesto, la pregunta de investigación es: ¿Cómo son los costos y plazos entre el ex ante y ex post en 22 PIP del GRM y GRA, en el periodo 2004-2020? La hipótesis planteada es que existe la percepción de que los periodos de ejecución de proyectos son mayores a los programados y los costos son superiores a los que fueron declarados viable. En ese sentido, la presente investigación analiza la ejecución de PIP que generen beneficios sociales en base a economías de escala, pues como explica (Vargas, 2007), esta relación sirve a más familias y personas.

Es preciso señalar que se entiende por economías de escala al proceso de un mayor volumen de producción con menores costos unitarios; al mismo tiempo, se relaciona con la elasticidad. Aquí, es preciso acelerar el crecimiento de la producción con los factores utilizados en el proceso económico. A partir de ello, se deriva que el principio de economía de escala, el valor del dinero en el tiempo, optimización de recursos y responsabilidad social son factores cuestionados en los gobiernos subnacionales analizados. La tarea es detectar plazos excesivos y la existencia de sobrecostos que, según Burgos y Vela (2015)multidrug resistanceassociated protein 1 (MRP1/ABCC1, son ocasionados por la escasa puntualidad en el desempeño de obras. 


\section{Material y métodos}

El tipo de investigación fue básica, por otra parte, fue necesario que exista una concatenación entre tipo, diseño, objetivos y métodos (Díaz y Calzadilla, 2016). Asimismo, fue no experimental, dado que se demostró que existen diferencias entre lo planteado en el periodo ex ante y lo que se efectivizó en el periodo ex post. Por último, la muestra escogida consistió en 22 proyectos de inversión (PI), 12 en Moquegua y 10 en la región Arequipa. Fueron elegidos debido a que estos explican, en promedio, entre el 60\% y $80 \%$ del presupuesto anual en los años que van del 2004 al 2020. También, son los proyectos que ejecutaron el GRM y el GRA en el periodo que, como se ve en cada uno de los años analizados, se repiten en promedio de 200 a 300 PI.

En este caso, se eligió una población de 22 proyectos de inversión, aun cuando se ha demostrado que 15 proyectos de inversión representan en promedio el 80\% del presupuesto en Moquegua y 10 representan el 60\% en Arequipa. Los criterios para elegir los proyectos analizados fueron dos: uno fue su ejecución en varios periodos y el segundo fue el incremento notorio de sus costos. Los 22 proyectos reunieron los requisitos mencionados y, finalmente, fueron tomados como muestra para la investigación.

Con un margen de error de $1 \%$, la técnica utilizada fue la observación, tal como la describe (Díaz \& Calzadilla, 2016), quienes orientan su utilización y su importancia. También, se recurrió al Portal de Transparencia del Ministerio de Economía y Finanzas (MEF). Adicionalmente, se tomaron los datos sobre la fecha de inicio de los proyectos de inversión, los tiempos y costos de ejecución y el cierre del año 2019 y 2020. Finalmente, se realizaron las pruebas de normalidad en seis distribuciones, la prueba de Wilcoxon para pruebas no paramétricas y las pruebas "t" para muestras relacionadas en el GRA.

\section{Resultados}

El análisis estadístico de la Tabla 6 muestra los plazos y costos de 10 proyectos: el PI con código 323143, el PI 2279710, el PI 246218, PI 249565, PI 2057380, PI 372773, el PI 2165385, PI 2111564, el PI 2290485 y el PI 2102290. En todos los casos, se comprueba que no tienen distribución normal. En la prueba Shapiro Wilk (SW), se obtuvo para costo ex ante Arequipa (0.000), costo ex post Arequipa (0.000), plazo ex ante Arequipa (o.033) y plazo ex post Arequipa (o.011). Al aplicar la prueba Wilcoxon, genera un P-valor de 0.007 que muestra la diferencia entre los costos del periodo anterior al efectivo. De la misma manera, los plazos propuestos y los plazos efectivos denotan que son diferentes en el sentido que los promedios 
Javier Pedro Flores Arocutipa, Jorge Jinchuña Huallpa, Rocío Claribel Cornelio Aira, Moisés Chacolla Soto, José Miguel de la Paz Ramos y Luís Enrique Fernández Sosa

van de 5.4 meses a 57.5 meses; por lo mismo, la prueba de Wilcoxon genera un p-valor de 0.005. En relación con los plazos, en la penúltima columna de la Tabla 6, se expone el incremento de tiempo en porcentajes y, en la última, se expone el exceso porcentual del costo.

\section{Tabla 6. Seguimiento de proyectos de inversión pública, Región Arequipa}

\begin{tabular}{cccccccc}
\hline $\mathbf{N}^{\circ}$ & $\begin{array}{c}\text { CUI de } \\
\text { inversiones }\end{array}$ & $\begin{array}{c}\text { Monto } \\
\text { (soles) }\end{array}$ & $\begin{array}{c}\text { Plazo } \\
\text { Ejec }\end{array}$ & $\begin{array}{c}\text { Monto } \\
\text { Actual } \\
\text { ejecución } \\
\text { (soles) }\end{array}$ & $\begin{array}{c}\text { meses de } \\
\text { ejecución }\end{array}$ & $\begin{array}{c}\text { Tiempo } \\
\text { (1) } \\
\text { exceso }\end{array}$ & $\begin{array}{c}\text { Costos } \\
\text { (1) } \\
\text { exceso }\end{array}$ \\
\hline 1 & 323143 & $46,094,512$ & 24 & $62,845,047$ & 52 & 116.7 & 36.34 \\
2 & 2279710 & $72,120,588$ & 24 & $90,946,659$ & 49 & 104.2 & 26.10 \\
3 & 246218 & $590,385,602$ & 17 & $652,652,270$ & 42 & 147.1 & 10.55 \\
4 & 249565 & $214,432,065$ & 18 & $627,536,702$ & 48 & 166.7 & 192.65 \\
5 & 2057380 & $48,800,168$ & 24 & $54,974,937$ & 150 & 525.0 & 12.65 \\
6 & 372773 & $1,199,270$ & 3 & $2,811,496$ & 11 & 266.7 & 134.43 \\
7 & 2165385 & $7,314,456$ & 9 & $7,285,106$ & 35 & 288.9 & -0.40 \\
8 & 2111564 & $34,414,668$ & 24 & $63,109,573$ & 55 & 129.2 & 83.38 \\
9 & 2290485 & $12,739,166$ & 6 & $16,392,957$ & 48 & 700.0 & 28.68 \\
10 & 2102290 & $2,990,669$ & 5 & $6,714,919$ & 85 & 1600.0 & 124.53 \\
\hline
\end{tabular}

Fuente: Elaboración propia con información de consulta amigable, consulta inversiones, (SSI - MEF)

Se debe mencionar que la sumatoria del volumen en PI del GRA, declarados costos viables, fueron de 1030 millones de soles. Al cierre del periodo de 2020 o a la fecha de culminación, este costo se incrementó a 1585 millones, mostrando una diferencia de 554 millones que sería el sobrecosto en soles corrientes, sin considerar el valor del dinero en el tiempo que resultaría mucho mayor. Lo mismo ocurre en el GRM, puesto que se tenía un costo viable de 127 millones que se incrementa en el tiempo a 220 millones de soles. Un sobrecosto de 92.6 millones. En la tabla 7, se presentan los costos y plazos de la región de Moquegua. 
Tabla 7. Costos y plazos mayores en PI del GRM

\begin{tabular}{cccccc}
\hline No & $\begin{array}{c}\text { Código } \\
\text { único de } \\
\text { inversiones }\end{array}$ & $\begin{array}{c}\text { Monto del } \\
\text { Estudio } \\
\text { Definitivo o } \\
\text { Expediente } \\
\text { Técnico }\end{array}$ & $\begin{array}{c}\text { Devengado } \\
\text { Acumulado } \\
\text { al 31 de } \\
\text { diciembre } \\
\text { del 2020 }\end{array}$ & $\begin{array}{c}\text { Plazo } \\
\text { Ejecución } \\
\text { Exp. Tec. } \\
\text { (días } \\
\text { calendario) }\end{array}$ & $\begin{array}{c}\text { Total, días } \\
\text { acumulados } \\
\text { a enero } \\
\mathbf{2 0 2 1}\end{array}$ \\
\hline 1 & 2022049 & 18630088 & 31601477 & 365 & 3559 \\
2 & 2109460 & 38258125 & 60619560 & 450 & 3784 \\
3 & 2159754 & 7482438 & 15516834 & 270 & 2572 \\
4 & 2087465 & 9726245 & 19537802 & 360 & 2970 \\
5 & 2159756 & 6282422 & 6570375 & 520 & 3006 \\
6 & 2091434 & 10441577 & 10107857 & 184 & 3032 \\
7 & 2159755 & 5451134 & 5180591 & 540 & 3013 \\
8 & 2160741 & 8793496 & 17300852 & 300 & 2542 \\
\hline 9 & 2164210 & 3322381 & 6127622 & 120 & 1851 \\
10 & 2166593 & 216834 & 6468756 & 180 & 1841 \\
11 & 2167234 & 698021 & 6795362 & 240 & 1166 \\
12 & 2188230 & 19586301 & 34228367 & 540 & 2421 \\
\hline
\end{tabular}

Fuente: Elaboración propia con información de (SSI - MEF)

En la Tabla 8, se comprueba seis distribuciones que tienen normalidad y dos que no, según la prueba SW. En la Tabla 9, se muestra la prueba Wilcoxon para pruebas no paramétricas. Esta enseña que hay diferencias entre costos ex ante y ex post en Arequipa (0.007) y Moquegua (0.006), aquí se incluye también la diferencia de plazos en Arequipa (0.0045). En la Tabla 10, se nota que sí hay disparidad de plazos; se utiliza la prueba " $t$ " para muestras relacionadas en el GRA. Se presenta que son distintos en términos de días, dado que la sig. (bilateral) es de o.ooo. En este caso, puede observarse que la media del plazo ex ante es de 339 días, mientras que la media del plazo actualizado es de 2307 días. Los datos ex ante y ex post son diferentes. 
Javier Pedro Flores Arocutipa, Jorge Jinchuña Huallpa, Rocío Claribel Cornelio Aira, Moisés Chacolla Soto, José Miguel de la Paz Ramos y Luís Enrique Fernández Sosa

814 Escasa eficiencia en costos y plazos en proyectos de inversión en Arequipa y Moquegua 2004-2020

\section{Tabla 8. Normalidad}

\begin{tabular}{|c|c|c|c|c|c|c|}
\hline & $\begin{array}{l}\text { Kolmogorov- } \\
\text { Smirnova }\end{array}$ & & & $\begin{array}{l}\text { Shapiro- } \\
\text { Wilk }\end{array}$ & & \\
\hline Moquegua-Arequipa & & $\mathrm{gl}$ & Sig. & & gl & Sig. \\
\hline Costo ex ante Moquegua & .261 & 12 & .023 & .826 & 12 & .019 \\
\hline Costo ex post Moquegua & .221 & 12 & .108 & .786 & 12 & .006 \\
\hline Plazo Moquegua ex ante & .140 & 12 &, $200^{*}$ & .929 & 12 & .365 \\
\hline Plazo ex post Moquegua & .129 & 12 &, $200^{*}$ & .973 & 12 & .939 \\
\hline Costo ex ante Arequipa & .367 & 10 & .000 & .607 & 10 & .000 \\
\hline Plazo ex ante Arequipa & .236 & 10 & .122 & .830 & 10 & .033 \\
\hline Costo ex post Arequipa & .404 & 10 & .000 & .614 & 10 & .000 \\
\hline Plazo ex post Arequipa & .327 & 10 & .003 & .792 & 10 & .011 \\
\hline $\begin{array}{l}\text { *. Esto es un límite } \\
\text { inferior de la significación } \\
\text { verdadera. }\end{array}$ & & & & & & \\
\hline
\end{tabular}

Tabla 9. Estadísticos de prueba

\begin{tabular}{lccc}
\hline & $\begin{array}{l}\text { Costo ex post } \\
\text { Arequipa - Costo } \\
\text { ex ante Arequipa }\end{array}$ & $\begin{array}{l}\text { Costo ex post } \\
\text { Moquegua - costo } \\
\text { ex ante Moquegua }\end{array}$ & $\begin{array}{c}\text { Plazo ex post Arequipa - } \\
\text { Plazo ex ante Arequipa }\end{array}$ \\
\hline $\mathrm{Z}$ & $-2,701 \mathrm{~b}$ & $-2,746 \mathrm{~b}$ & $-2,805^{\mathrm{b}}$ \\
$\begin{array}{l}\text { Sig. asintótica } \\
\text { (bilateral) }\end{array}$ & .007 & .006 & .0045 \\
\hline
\end{tabular}

a. Prueba de rangos con signo de Wilcoxon

b. Se basa en rangos negativos.

Tabla 10. Diferencias emparejadas

\begin{tabular}{lllllll}
\hline & \multicolumn{5}{c}{$\begin{array}{l}\text { 95\% de intervalo } \\
\text { de confianza de la } \\
\text { diferencia }\end{array}$} \\
& $\begin{array}{l}\text { Media } \\
\text { de error } \\
\text { estándar }\end{array}$ & Inferior & Superior & & & \\
& 185.7 & -2377.0 & -1559.5 & -10.6 & 11 & .000 \\
\hline $\begin{array}{l}\text { Plazo Moquegua ex ante - } \\
\text { plazo ex post Moquegua }\end{array}$ & & & & & & \\
\hline
\end{tabular}

Fuente: Elaboración propia. 


\section{Discusión}

Es notorio que el PBI de Arequipa incrementó en 3.46\% anual promedio y el de Moquegua solamente creció en 0.9\% entre los años 2007 y 2020. Al respecto, se analiza lo explicado por (Villegas, 2018), quien probó que la ejecución puntual de los proyectos repercute en el crecimiento económico de un distrito. Ante el crecimiento mínimo expuesto y los plazos postergados, se evidencia una situación preocupante en ambas regiones.

La percepción es hacer crecer la renta nacional per cápita, la cual se interpreta como calidad de vida. La inversión pública y privada efectiva impulsan hacia este objetivo. En ese sentido el objetivo es el desarrollo económico que coincide con los resultados de (Duarte \& Ruíz, 2015). Pero para lograrlo se tienen elementos perturbadores; los mayores costos, y allí coindice con la propuesta de Balcázar (2015), citado por (Fernández, 2018), aunque ellos agregan el saneamiento de los terrenos donde se pretende erigir las infraestructuras. Este problema es notorio también en los gobiernos subnacionales de las regiones analizadas. (Liñán, 2019) también concluye que otro factor, además de los mencionados, son las ampliaciones. De manera precisa, (Fernández, 2018) indica que las buenas compras de insumos ayudan a la logística de productividad; sin embargo, cuando no se cumple el precepto, generan laudos arbitrales que terminan favoreciendo en su mayor parte al proveedor.

Hay sectores por función que se caracterizan por los atrasos en inicio y ejecución de obras. Sobre ello, (Arela, 2020) ha señalado que estos son los sectores de transporte, educación y salud; al respecto (Romero, 2016), en su análisis, agrega el sector agricultura.

En tal sentido, se insiste en la política de gestión por resultados, y un requisito es el cumplimiento de la programación. Aun cuando los funcionarios lo saben, se observa que la función pública regional no está comprometida, y así también lo señala (Burgos \& Vela, 2015), (Almanza, 2018) además de (Porras \& Diaz, 2015), quienes agregan que inclusive el cálculo matemático no se valora. Otro elemento en el análisis son los indicios de corrupción, así lo manifiesta la contraloría de (Shack et al., 2020), quienes han observado que desde el perfil del proyecto se insinúan marcas y proveedores, de igual forma lo advierten (Gordo, 2017).

Como se mencionó anteriormente, para iniciar u operar un PI, es ideal buscar el menor costo de los factores de producción. En ese sentido, se coincide con la apreciación de (Huaquisto, 2016), quien aduce que aquellos factores son la eficiencia y la supervisión; de manera similar (Prado, 2015) sostiene que estas son claves para lograr la óptima producción y los menores costos y plazos. 
Javier Pedro Flores Arocutipa, Jorge Jinchuña Huallpa, Rocío Claribel Cornelio Aira, Moisés Chacolla Soto, José Miguel de la Paz Ramos y Luís Enrique Fernández Sosa

816 Escasa eficiencia en costos y plazos en proyectos de inversión en Arequipa y Moquegua 2004-2020

Es un hecho de la probación que los conceptos metas, tiempo y recursos para medir eficiencia y eficacia no son aplicados por la función pública, aquí se coincide con (Galván \& García, 2019). Como señalan (Sapag \& Sapag, 2008) y (Dueñas, 2017), si el análisis fuera por el valor del dinero en el tiempo, se tendría el valor actual neto social por debajo de cero, y una tasa de retorno social por debajo del costo del capital, con lo cual se confirma la pérdida de valor social.

Para realizar un análisis de costo beneficio, los flujos deben ser sometidos a un costo de oportunidad, en este caso del capital, y se refiere a lo que perdería por elegir el proyecto, desde la perspectiva más rentable. Aquí la pérdida es clara, el costo del dinero por no realizar un proyecto en el plazo estipulado y la repercusión social que conlleva. Es peor iniciar un proyecto en el año 1 y dejarlo el año 2 y 3 paralizados para retomarlo el año cuatro, y así sucesivamente. Esto crea un grave perjuicio a la economía de la región. Como señala (Blondet, 2013), el costo de no hacer obras en sus plazos y costos, agregando la corrupción, afecta el desarrollo del país. De esta manera, se muestra que las inversiones ejecutadas en los plazos adecuados garantizan el crecimiento económico.

Si se liquidaran las obras (evaluación ex post) en su debido tiempo, se lograría información para transparentar y corregir las deficiencias que se han probado, consecuentemente, se lograrían los objetivos con mejores resultados. Aquí se coincide con (Beltrán, 2010), quien señalar que al lograr el beneficio social, se traduce en mayor incremento estándar del PBI y calidad de vida.

\section{Conclusiones}

Se ha demostrado que existe escasa eficiencia en costos y plazos en 22 PIP de los GR de Moquegua y Arequipa, los cuales generaron sobrecostos por 646 millones de soles y un promedio de plazos excesivos de 1968 días. La prueba estadística verificó las diferencias. La escasa eficacia en proyectos de inversión se relaciona de manera directa con los informes de la contraloría, en base a la extrapolación generado por Nelson Shack, la corrupción le "costo" al departamento de Arequipa 9716 millones y a Moquegua 2470 millones de soles y en general al sur peruano 36 mil 296 millones de soles en la última década (2011-2020).

Moquegua Y Tumbes Lideran con funcionarios públicos con Presunta responsabilidad Civil Y Penal Por 100 Mil Habitantes. Se entiende en alguna forma del porque los retrasos en las obras, son mayores plazos y costos.

El informe de la contraloría general, ha sentenciado que el $30 \%$ del presupuesto anual y el $3 \%$ del PBI es lo que cuestan los actos de corrupción 
en el Perú, cada uno de los años en la última década. Para ello se ha calculado el valor del PBI regional y se ha encontrado cómo en las regiones del sur peruano, se afecta a su riqueza. Son 1.33 veces el presupuesto anual de inversiones de Arequipa, y 3 veces el presupuesto de inversiones de Moquegua. Por ello cuando se señala que la corrupción afecta al crecimiento económico, se tiene toda la razón.

De todas maneras, el soborno, que es pedir o recibir, es conocido en el argot penal como cohecho. Son los que terminan elevando los costos que pagan los peruanos a través de los impuestos que recauda el estado. Y con obras que no son ejecutadas o con plazos excesivos donde los servicios no llegan a los ciudadanos. Ahí está la infraestructura de salud, débil, fallida, que permitió que fallezcan 200 mil peruanos y que llevo dolor a las familias de los peruanos. La corrupción generó pérdidas por 608 soles a cada arequipeño y 1165 a cada moqueguano en el año 2020.

\section{Referencias Bibliográficas}

ALMANZA PAREDES, Cinthia Yurema. 2018. "Administración de proyectos de inversión pública ejecutados en la gestión gubernamental de la Municipalidad Provincial del Cusco 2015-2016". Tesis de grado de Maestría en Gestión Públcia. Universidad César Vallejo. Disponible en línea. En: https://repositorio.ucv.edu.pe/browse?type=author\&value= Almanza+Paredes\%2C+Cinthia+Yurema\&locale-attribute=es. Fecha de consulta: 05/06/2020.

ARELA BOBADILLA, Ronal. 2020. ¿Cómo se gasta el presupuesto de proyectos de inversión pública en Arequipa? Un análisis con técnicas de machine learning no supervisado. Informe del Centro de Estudios en Economía y Empresa. Universidad Católica San Pablo. Disponible en Línea. En: https://ucsp.edu.pe/wp-content/uploads/2020/11/Como-se-gastael-presupuesto-de-proyectos-de-inversion-publica-en-Arequipa-Nov2020-Ronal-Arela-CEEE-UCSP.pdf. Fecha de consulta: 15/10/2020.

BELTRÁN, Arlette. 2010. Balance de la inversión pública: Avances y desafíos para consolidar la competitividad y el bienestar de la población. Apuntes, $67,28$.

BLONDET, Cecilia. 2013. Reseña del libro "historia de la corrupción en el perú" de Alfonso Quiroz. En Revista Argumentos. Edición n ${ }^{\circ}$ 2, Mayo 2013. Disponible en línea. En: https://argumentos-historico.iep.org. pe/articulos/resena-del-libro-historia-de-la-corrupcion-en-el-peru-dealfonso-quiroz/. Fecha de consulta: 23/08/2020. 
Javier Pedro Flores Arocutipa, Jorge Jinchuña Huallpa, Rocío Claribel Cornelio Aira, Moisés Chacolla Soto, José Miguel de la Paz Ramos y Luís Enrique Fernández Sosa

818 Escasa eficiencia en costos y plazos en proyectos de inversión en Arequipa y Moquegua 2004-2020

BURGOS MARÍN, Mateo. VELA AVILA, Daniel. 2015. "Análisis de las causas del incumplimiento de la programación en las obras civiles". Trabajo de grado. Universidad Militar Nueva Granada. Disponible en línea. En: https://repository.unimilitar.edu.co/bitstream/ handle/10654/7285/BurgosMarinMateo.2015.VelaAvilaDaniel.2015. pdf?sequence=1\&isAllowed=y. Fecha de consulta: 12/01/2021.

DÍAZ NARVÁEZ, Victor Patricio., CALZADILLA NÚÑEZ, Aracelis. 2016. "Artículos científicos, tipos de investigación y productividad científica en las Ciencias de la Salud". Revista Ciencias de La Salud, 14(1), 115121. https://doi.org/10.12804/revsalud14.01.2016.10. Disponible en Línea. En: https://revistas.urosario.edu.co/index.php/revsalud/article/ view/4597. Fecha de consulta: 21/03/2021.

DUARTE, Tito; RUIZ TIBANA, Myriam. 2015. "Losproyectos de desarrollo: la inversión pública y la inversión privada" En: Scientia et Technica. Vol. 20, No. 02, pp. 134-139.

DUEÑAS, Alejandro. 2017. “El Sistema Nacional deInversión Públicayel Proceso Presupuestal en el Gobierno Regional de Madre de Dios -2017”. Tesis de grado de maestría. Universidad César Vallejo. Disponible en línea. En: http://repositorio.ucv.edu.pe/bitstream/handle/UCV/20052/dueñas_ da.pdf? sequence=1\&isAllowed=y. Fecha de consulta: 16/04/2021.

FERNÁNDEZ REINA, Enith Consuelo. 2018. "Factores asociados al cumplimiento en la ejecución de los proyectos de inversión pública en una obra de infraestructura pública". Tesis de grado para optar el grado de Doctor en Gestión Pública y Gobernabilidad. Universidad César Vallejo. Disponible en línea. En: https://repositorio.ucv. edu.pe/bitstream/handle/20.500.12692/19133/Fernández_REC. pdf? sequence $=4 \&$ isAllowed $=y$. Fecha de consulta:12/06/2020.

GALVÁN VELA, Esthela. GARCÍA RUÍZ, Jesús Enrique. 2019. La eficiencia y su relación con el éxito de un proyecto según administradores de proyectos en centros de investigación. Fides et Ratio - Revista de Difusión cultural y científica de la Universidad La Salle en Bolivia, 17(17), 193-214. Disponible en línea. En: http://www.scielo.org.bo/scielo. php?script=sci_arttext\&pid=S2071-081X2019000100010\&lng=es\&tln $\mathrm{g}=$ es. Fecha de consulta: 8/12/2020.

GORDO BARREIRO, Eduar Mauricio. POTES LÓPEZ Johana Andrea. VARGAS QUIMBAYA, .Luis. 2017. "Factores que ocasionan retrasos en obras civiles en Empresas Publicas de Neiva”. Trabajo para optar por el título de especialista en auditoría de proyectos. Universidad Santo Tomas de Colombia. Disponible en línea. En: https://repository. usta.edu.co/bitstream/handle/11634/10740/Johana\%20Potes-2017. pdf?sequence=1\&isAllowed=y. Fecha de consulta: 30/11/2020. 
HUAQUISTO CÁCERES, Samuel. 2016. "Análisis de eficiencia en proyectos de inversión Pública: un estudio de caso en proyectos ejecutados por Administración directa". Revista de Investigaciones Altoandinas Journal of High Andean Research, 18(1), 61. https://doi.org/10.18271/ ria.2016.179. Disponible en línea. En: https://dialnet.unirioja.es/servlet/ articulo? codigo $=5399051$. Fecha de consulta: 09/05/2021.

INSTITUTO NACIONAL DE ESTADÍSTICA E INFORMÁTICA - INEI. 2021. Economía. Principales indicadores macroecómicos. Documento oficial. Disponible en línea. En: https://www.inei.gob.pe/estadisticas/indicetematico/economia/. Fecha de consulta: 04/08/2021.

LATINOBARÓMETRO. 2021. Opinión pública latinoamericana. Disponible en línea. En: https://www.latinobarometro.org/lat.jsp. Fecha de consulta: 03/07/2021.

LIÑÁN IZAGUIRRE, Francisco Manuel. 2019. "Efectos de las ampliaciones de plazo en las obras públicas”. Tesis para optar grado de maestría en Gestión Publica. Universidad César Vallejo. Disponible en línea. En: https:// repositorio.ucv.edu.pe/bitstream/handle/20.500.12692/26072/ Li\%C3\%B1an_IFM.pdf?sequence=1\&isAllowed=y. Fecha de consulta: 02/10/2020.

MINISTERIO DE ECONOMÍA Y FINANZAS, DIRECCIÓN GENERAL DE PROGRAMACIÓN MULTIANUAL DE INVERSIONES (MEF - DGPMI). 2019. Guía General para la Identificación, Formulación y Evaluación de Proyectos de Inversión 2019. Invierte.Pe, 200. Disponible en línea. . En: https://www.dnp.gov.co/Programas/ Inversionesyfinanzasp\%FAblicas/6Metodolog\%EDas.aspx. Fecha de consulta: 09/09/2021.

MINISTERIO DE ECONOMÍA Y FINANZAS - DIRECCIÓN GENERAL DE PROGRAMACIÓN MULTIANUAL DE INVERSIONES (MEF DGPMI). 2021. Seguimiento de la Ejecución Presupuestal (Consulta amigable). Disponible en línea. En: https://www.mef.gob.pe/ es/?option=com_content\&language=es-ES\&Itemid=100944\&lang=esES\&view=article\&id=504. Fecha de consulta: 07/07/2021.

SÁNCHEZ GARCÍA, Edgar German. 2020. "Reflexiones sobre el debido proceso en las Constituciones de América Latina” En: Cuestiones Políticas. Vol. 37, No. 64, pp. 44-52. Disponible en línea. En: https://doi.org/10.46398/ cuestpol.3764.02. Fecha de consulta: 28/08/2021.

SAPAG CHAIN, Nassir. SAPAG CHAIN, Reinaldo. 2008. Preparación y evaluación de proyectos. Printed in Colombia. Mc Graw Hill (Quinta, Vol. 53, Issue 9). Mc Graw Hill. Disponible en líne. En: https://untdfproyectos. 
Javier Pedro Flores Arocutipa, Jorge Jinchuña Huallpa, Rocío Claribel Cornelio Aira, Moisés Chacolla Soto, José Miguel de la Paz Ramos y Luís Enrique Fernández Sosa

820 Escasa eficiencia en costos y plazos en proyectos de inversión en Arequipa y Moquegua 2004-2020

files.wordpress.com/2018/04/sapag-2008-preparacion-y-evaluacionde-proyectos.pdf. Fecha de consulta: 19/03/2021.

SISTEMA DE SEGUIMIENTO DE INVERSIONES, MINISTERIO DE ECONOMÍA Y FINANZAS (SSI - MEF). 2021. Consulta en línea. Invierte.Pe. Disponible en línea. En: https://ofi5.mef.gob.pe/ssi/ssi/ Index. Fecha de consulta: 06/04/2021.

PRADO ESPINO, Nancy. 2015. "Auditoria de cumplimiento y su incidencia en la detección de fraudes en la ejecución de peroyectos de inversión por administración directa en el Gobierno Regional de Ayacucho-2014”. Tesis de Grado de Maestría. Universidad Católica Los Angeles de Chimbote. Disponible en línea. En: http://repositorio.uladech.edu.pe/bitstream/ handle/123456789/881/AUDITORIA_DE_CUMPLIMIENTO_ FRAUDE_PRADO_ESPINO_NANCY.pdf? sequence $=1 \&$ isAllowed $=\mathrm{y}$. Fecha de consulta: 09/08/2021.

PORRAS MOYA, David Alejandro, DIAZ MONTERO, Jhon. Edinson. 2015. "La planeación y ejecución de las obras de construcción dentro de las buenas prácticas de la administración y programación (proyecto Torres de la 26-Bogotá)". Tesis para optar título de Ingeniero Civil. Universidad Católica de Colombia. Disponible en línea. En: https://repository. ucatolica.edu.co/bitstream/10983/2951/4/. Fecha de consulta: 03/02/2021.

PROETICA. 2021. Transparency International. Casos emplematícos. Disponible en línea. En: https://www.proetica.org.pe/casos-emblematicos/. Fecha de consulta: 06/06/2021.

ROMERO RODRIGUEZ, Carlos Alexis. 2016. "Analisis de la ejecución de la inversión pública y su incidencia en la calidad de vida de la población: región Libertad periodo 2009-2014". Tesis de grado para optar el grado de Maestro en Ciencias Económicas. Universidad Nacional de Trujillo. Disponible en línea. En: https://dspace.unitru.edu.pe/ bitstream/handle/UNITRU/2436/Tesis de Maestría_Carlos Alexis Romero Rodríguez.pdf?sequence $=1$ \&isAllowed $=y$. Fecha de consulta: 02/09/2020.

SHACK YALTA, Nelson; PEREZ PINILLOS, Jeniffer; PORTUGAL LOZANO, Luís. 2020. Cálculo del tamaño de la corrupción y la inconducta funcional en el Perú: Una aproximación exploratoria. Documento de Política en Control Gubernamental. Contraloría General de la República. Lima, Perú. Disponible en línea. En: https://doc.contraloria.gob.pe/estudiosespeciales/documento_trabajo/2020/Calculo_de_la_Corrupcion_en_ el_Peru.pdf. Fecha de consulta: 07/08/2021. 
VARGAS SÁNCHEZ, Gustavo. 2007. Vargas Sánchez, Gustavo. (2007). "La nueva microeconomía dinámica" En: Investigación económica. Vol. 66, No. 262, pp. 171-204. Disponible en línea. En: http://www.scielo.org. $\mathrm{mx} /$ scielo.php?script=sci_arttext\&pid=S0185-16672007000400171\&ln $\mathrm{g}=\mathrm{es} \&$ tlng=es . Fecha de consulta: 01/12/2020.

VILLEGAS LUJÁN, Ermers. 2018. "Factores que condicionan la eficiencia de los proyectos de inversión pública y su influencia en el desarrollo sostenible del distrito de Sanagorán, 2017". Tesis de grado para optar el grado de Maestro en Gestión Pública. Universidad César Valljo. Disponible en línea. En: https://repositorio.ucv.edu.pe/bitstream/ handle/20.500.12692/17699/villegas_le.pdf?sequence=1. Fecha de consulta: $02 / 02 / 2021$. 

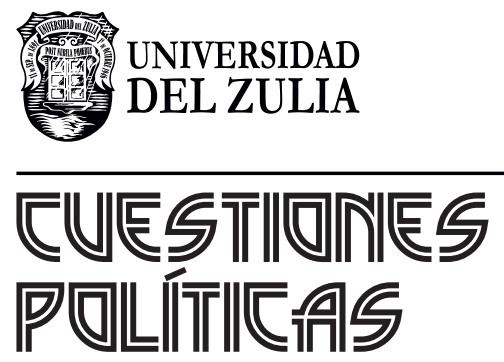

Vol. 39 N $^{\circ} 71$

Esta revista fue editada en formato digital y publicada en diciembre de 2021, por el Fondo Editorial Serbiluz, Universidad del Zulia. Maracaibo-Venezuela 